\title{
ON THE GAPS IN THE SPECTRUM ASSOCIATED WITH HILL'S EQUATION
}

\author{
M. S. P. EASTHAM
}

1. Let $q(x)$ be a real-valued, piecewise continuous, periodic function with period $a$ and let $S$ denote the spectrum associated with the equation

$$
\psi^{\prime \prime}(x)+\{\lambda-q(x)\} \psi(x)=0
$$

holding for $-\infty<x<\infty$. Any gap in $S$ with midpoint $\mu$ has length not exceeding

$$
2\left(\frac{1}{a} \int_{0}^{a} q^{2}(x) d x\right)^{1 / 2}
$$

if $\mu \geqq 0$. This was first proved by Putnam [3] and later obtained as a special case of a more general result [2, Theorem 3]. Putnam queried the necessity of the condition $\mu \geqq 0$ and raised the question of whether all gaps in $S$ do not exceed (1.2) in length. In this connexion, he pointed out that, if $q(x)$ has mean value zero, i.e. if

$$
\int_{0}^{a} q(x) d x=0
$$

then all gaps in $S$ do not exceed (1.2) in length if

$$
\int_{0}^{a} q^{2}(x) d x \leqq 256 a^{-3}
$$

inasmuch as that this condition automatically implies that $\mu \geqq 0$ for all gaps. Actually, Putnam worked with $a=1$, but the results for general $a$ are obtained by the transformation $x=a t$.

In this paper I answer Putnam's question in the negative in $\$ 2$ by constructing a $q(x)$ satisfying (1.3) such that the first gap in $S$ has length exceeding (1.2). I mention here for comparison with (1.4) that my $q(x)$ satisfies

$$
\int_{0}^{a} q^{2}(x) d x>2{ }^{10} \pi^{4} 256 a^{-3} .
$$

Then, in $\S 3$, I give an extension of the result concerning (1.2).

Received by the editors July 13, 1968. 
2. Let $\mu_{n}$ and $\Lambda_{n}$ be the eigenvalues of (1.1) for the interval $0 \leqq x \leqq a$ with the boundary conditions

$$
\psi(0)=-\psi(a), \quad \psi^{\prime}(0)=-\psi^{\prime}(a)
$$

and

$$
\psi(0)=\psi(a)=0
$$

respectively. Then the first gap in $S$ is the interval $\left(\mu_{0}, \mu_{1}\right)$, and our aim is to construct a $q(x)$ for which

$$
\mu_{0}+2\left(\frac{1}{a} \int_{0}^{a} q^{2}(x) d x\right)^{1 / 2}<\mu_{1} .
$$

Let $\alpha, b, c$ be positive numbers with $2 b+2 c \leqq a$ and let

$$
\begin{aligned}
q(x) & =-\alpha & & 0 \leqq x \leqq b, \\
& =0 & & b<x \leqq b+c, \\
& =\alpha & & b+c<x \leqq 2 b+c, \\
& =0 & & 2 b+c<x \leqq a .
\end{aligned}
$$

Clearly (1.3) is satisfied. We now obtain estimates for $\mu_{0}$ and $\mu_{1}$ and impose conditions on $\alpha, b, c$ as we go along. The simplest way to deal with $\mu_{1}$ is to use the inequality $\mu_{1} \geqq \Lambda_{0}[1$, p. 215 (3.15)]. Let

$$
\alpha b^{2} \leqq \frac{1}{4} \pi^{2}
$$

Then, if $\Lambda_{0}^{\prime}$ corresponds to $\Lambda_{0}$ in the problem with $q(x)$ replaced by $q_{1}(x)$, where

$$
\begin{aligned}
q_{1}(x) & =-\alpha & & (0 \leqq x \leqq b), \\
& =0 & & (b<x \leqq a),
\end{aligned}
$$

we have $\Lambda_{0}^{\prime}>0[4, \S 5.5]$. Since $q(x) \geqq q_{1}(x), \Lambda_{0} \geqq \Lambda_{0}^{\prime}$ and therefore

$$
\mu_{1}>0 \text {. }
$$

Next,

$$
\mu_{0}=\inf \left(\int_{0}^{a}\left[\left\{f^{\prime}(x)\right\}^{2}+q(x) f^{2}(x)\right] d x / \int_{0}^{a} f^{2}(x) d x\right),
$$

the inf being taken over all continuous $f(x)$ with piecewise continuous derivative such that $f(0)=-f(a)$. Choose $f(x)$ to be the function whose graph consists of straight line segments joining the points $(0,1),(b, 1),(b+c, 0),(a-c, 0),(a,-1)$. Then, as is easily verified, 


$$
\mu_{0} \leqq \frac{2 / c-\alpha b}{2 c / 3+b}=\frac{1}{b^{2}} \frac{2 d-\alpha b^{2}}{2 / 3 d+1},
$$

where $d=b / c$. Hence, by (2.4), (2.1) is satisfied if

$$
\frac{1}{b^{2}} \frac{2 d-\alpha b^{2}}{2 / 3 d+1}+2\left(2 \alpha^{2} b / a\right)^{1 / 2} \leqq 0
$$

i.e. if $a^{1 / 2} \geqq 2 \sqrt{2} \alpha b^{5 / 2}(2 / 3 d+1) /\left(\alpha b^{2}-2 d\right)$. The best choice of $d$ would be the one between 0 and $\frac{1}{2} \alpha b^{2}$ which minimizes the right-hand side. A convenient choice, however, is $d=\frac{1}{4} \alpha b^{2}$. Thus we choose

$$
c=4 / \alpha b \text {. }
$$

This gives $a \geqq 32 b\left(8 / 3 \alpha b^{2}+1\right)^{2}$. Hence, by (2.3), we can take

$$
\begin{aligned}
\alpha b^{2} & =\frac{1}{4} \pi^{2}, \\
a & =32 b\left(32 / 3 \pi^{2}+1\right)^{2} .
\end{aligned}
$$

Reversing the steps, given $a$, we choose $b$ so that (2.7) holds and then $\alpha$ and $c$ so that (2.6) and (2.5) hold. Then (2.2) gives the required $q(x)$.

Comparing our result with (1.4), we have

$$
\int_{0}^{a} q^{2}(x) d x=2 \alpha^{2} b=2\left(\frac{1}{4} \pi^{2}\right)^{2} b^{-3}
$$

which, by (2.7) gives (1.5) since $32 / 3 \pi^{2}>1$. No doubt the multiple of $a^{-3}$ which occurs here could be reduced to some extent at the cost of more complicated analysis.

3. We return now to (1.1) with general $q(x)$ satisfying (1.3). If $q(x)$ has the Fourier series $\sum c_{r} \exp (2 \pi r x i / a)$, then the expression (1.2) in Putnam's result is

$$
2\left(2 \sum_{1}^{\infty}\left|c_{r}\right|^{2}\right)^{1 / 2}
$$

Our extension is as follows:

THEOREM. For any integer $N \geqq 1$, a gap in $S$ with midpoint $\mu$ has length not exceeding

$$
2\left(2 \sum_{N+1}^{\infty}\left|c_{r}\right|^{2}\right)^{1 / 2}+\frac{2 \pi}{a}\left(2 \sum_{1}^{N} r^{2}\left|c_{r}\right|^{2} /\left(\mu-2 \sum_{1}^{N}\left|c_{r}\right|\right)\right)^{1 / 2}
$$

if $\mu>2 \sum_{1}^{N}\left|c_{r}\right|$. 
We use the result that a gap in $S$ with midpoint $\mu$ has length not exceeding

$$
2 \liminf _{m \rightarrow \infty}\left\|\{q(x)-\mu\} f_{m}(x)-f_{m}^{\prime \prime}(x)\right\|
$$

for any sequence $\left\{f_{m}\right\}$ such that $\left\|f_{m}\right\|=1, f_{m}$ converges weakly to zero in $L^{2}(-\infty, \infty)$, and $f_{m}(x)$ has compact support and a continuous second derivative. Here, the norm is that of the complex space $L^{2}(-\infty, \infty)[3$, inequality (8)]; [2, (3.3)]. Let $g(x)$ be any fixed function defined for $0 \leqq x \leqq 1$ such that

$$
\begin{array}{r}
g(0)=0, \quad g(1)=1, \quad g^{\prime}(0)=g^{\prime \prime}(0)=g^{\prime}(1)=g^{\prime \prime}(1)=0, \\
0 \leqq g(x) \leqq 1
\end{array}
$$

and let

$$
Q(x)=\int_{0}^{x}\left(\mu-\sum_{-N}^{N} c_{r} \exp (2 \pi r t i / a)\right)^{1 / 2} d t .
$$

Now define $f_{m}(x)=B_{m} e^{i Q(x)} h_{m}(x)$, where

$$
\begin{aligned}
h_{m}(x) & =1 & & |x| \leqq m a-1, \\
& =g(m a-|x|) & & m a-1<|x| \leqq m a, \\
& =0 & & |x|>m a,
\end{aligned}
$$

and $B_{m}$ is the normalization constant. Now $Q(x)$ is real-valued if $\mu>2 \sum_{1}^{N}\left|c_{r}\right|$ and it is then easy to verify that

$$
\begin{gathered}
\left|f_{m}(x)\right| \leqq B_{m}, \\
B_{m} \sim(2 m a)^{-1 / 2} \quad(m \rightarrow \infty),
\end{gathered}
$$

and that $f_{m}(x)$ satisfies the above conditions for (3.3) (cf. [2, §2]). We have

$$
\begin{aligned}
\{q(x)-\mu\} & f_{m}(x)-f_{m}^{\prime \prime}(x) \\
& =\left\{q(x)-\mu+Q^{\prime 2}(x)\right\} f_{m}-2 i B_{m} e^{i Q} Q^{\prime} h_{m}^{\prime}-i Q^{\prime \prime} f_{m}-B_{m} e^{i Q} h_{m}^{\prime \prime} \\
& =u_{1}(x)+u_{2}(x)+u_{3}(x)+u_{4}(x),
\end{aligned}
$$

say. It is easy to check that $\left\|u_{2}(x)\right\| \rightarrow 0$ and $\left\|u_{4}(x)\right\| \rightarrow 0$ as $m \rightarrow \infty$. By (3.4) and (3.5),

$$
\begin{aligned}
\left\|u_{1}(x)\right\| & \leqq B_{m}\left\{2 m \int_{0}^{a}\left(q(x)-\sum_{-N}^{N} c_{r} \exp (2 \pi r x i / a)\right)^{2} d x\right\}^{1 / 2} \\
& =B_{m}(2 m a)^{1 / 2}\left(2 \sum_{N+1}^{\infty}\left|c_{r}\right|^{2}\right)^{1 / 2}
\end{aligned}
$$


and

$$
\begin{aligned}
& \left\|u_{3}(x)\right\| \leqq B_{m}\left(2 m \int_{0}^{a}\left\{Q^{\prime \prime}(x)\right\}^{2} d x\right)^{1 / 2} \\
& \quad \leqq B_{m}(2 m a)^{1 / 2} \frac{1}{2}\left(\mu-2 \sum_{1}^{N}\left|c_{r}\right|\right)^{-1 / 2}\left(2 \sum_{1}^{N}\left(2 \pi r\left|c_{r}\right| / a\right)^{2}\right)^{1 / 2} .
\end{aligned}
$$

The theorem now follows from (3.3), (3.6), (3.7), and (3.8).

Putnam's proof of (3.1) was in essence the case $N=0$ of the above proof. We note that (3.2) is certainly not greater than (3.1) if $\mu$ is large enough. Indeed, (3.2) gives a new proof that the gap with midpoint $\mu$ tends to zero in length as $\mu \rightarrow \infty$. To see this, for any $\epsilon>0$, we choose $N$ so that the first term in (3.2) is less than $\frac{1}{2} \epsilon$ and then $\mu$ so that the second term is less than $\frac{1}{2} \epsilon$. Thus (3.2) is less than $\epsilon$ if $\mu \geqq \mu(\epsilon)$.

\section{REFERENCES}

1. E. A. Codgington and N. Levinson, Theory of ordinary differential equations, McGraw-Hill, New York, 1955.

2. M. S. P. Eastham, On the limit points of the spectrum, J. London Math. Soc. 43 (1968), 253-260.

3. C. R. Putnam, On the gaps in the spectrum of the Hill equation, Quart. Appl. Math. 11 (1953), 496-498.

4. E. C. Titchmarsh, Eigenfunction expansions, 2nd ed., Clarendon Press, Oxford, 1962.

The University, Southampton, England 